\title{
ReVIEW
}

\section{Clinical features and diagnosis of multiple myeloma}

\author{
Michael J Wong, MD', Trudy Taylor, MD FRCPC ${ }^{2}$ \\ 'Department of Anesthesiology, Pain Management \& Perioperative Medicine, \\ Dalhousie University \\ ${ }^{2}$ Department of Medicine, Division of Rheumatology, Dalhousie University
}

\section{Clinical Vignette}

EM, an 85 year-old female, was admitted to the Medical Teaching Unit with a one-week history of confusion. In the Emergency Department, she was disoriented and later became somnolent. During the month prior to admission, she had experienced progressive mid-back pain, and had been diagnosed with a T8 compression fracture.

Laboratory investigations showed a hemoglobin of $81 \mathrm{~g} / \mathrm{L}$ with mean corpuscular volume of $101 \mathrm{fL}$. Rouleaux formations were seen on peripheral smear. EM had elevated creatinine $(133 \mathrm{mmol} / \mathrm{L})$, urea $(11.2$ $\mathrm{mmol} / \mathrm{L})$, and ionized calcium $(1.97 \mathrm{mmol} / \mathrm{L})$; however, parathyroid hormone levels were normal, as were iron studies, vitamin B12, folate, and thyroid stimulating hormone (TSH).

Urine culture revealed Escherichia coli bacteriuria, which was treated with ceftriaxone. Pamidronate was administered for hypercalcemia. Early into the admission, she became fluid overloaded and required diuresis, while simultaneously receiving intravenous fluids for her hypercalcemia.

Multiple myeloma was considered as the cause of EM's constellation of symptoms, so a serum protein electrophoresis was performed, revealing an IgA monoclonal protein spike. Free light chain analysis showed an increase in free kappa light chains (7.69 $\mathrm{mg} / \mathrm{L}$ ) with a markedly elevated kappa/lambda ratio of 157.5 .

\section{Introduction}

Multiple myeloma (MM) refers to a malignant B-lymphocyte disorder characterized by proliferation of a single clone of plasma cells, and production of a monoclonal protein (M-protein). ${ }^{1,2}$ Also known as plasma cell myeloma, plasmacytic myeloma, myelomatosis, or Kahler's disease, the term MM can be misleading since plasma cells are of lymphoid lineage and not myeloid; rather, the name reflects involvement of the myelum or bone marrow, where plasma cells reside. ${ }^{1,2}$ Malignant transformation of plasma cells typically occurs in multiple bone sites, and may form masses capable of advancing locally or involving distant organs through lymphatic or hematogenous spread. ${ }^{3}$ The cause of MM remains uncertain, ${ }^{4}$ and it continues to be incurable, with almost all patients eventually developing treatment-resistant disease. ${ }^{5}$

The diagnosis of MM is made on the basis of a myeloma-defining event (e.g., end-organ damage, predictive biomarkers) and bone marrow biopsy showing monoclonal plasma cells. However, in the absence of significant bone marrow plasma cells, biopsy-confirmed presence of any localized proliferation of plasma cells (plasmacytoma) may suffice. ${ }^{6}$

\section{Epidemiology}

MM accounts for approximately $10 \%$ of hematologic cancers, $1 \%$ of all cancer diagnoses, and $1 \%$ of all cancer deaths. ${ }^{1,7}$ In Canada, around 2700 new cases of MM are diagnosed each year. ${ }^{8}$ The disease has a slight preponderance for males, and is significantly more common in Blacks compared to Caucasians, while Asians have a lower incidence. ${ }^{9}$ The median age at diagnosis is 62 years, with only $2 \%$ of cases diagnosed before 40 years. ${ }^{10}$ Median survival with conventional treatment is about four years, but median survival can be extended to five to seven years with high-dose treatment and autologous stem-cell transplantation., 2,10

In many patients, $M M$ is preceded by a pre-malignant stage called monoclonal gammopathy of undetermined significance (MGUS). ${ }^{7}$ MGUS has a $1 \%$ annual risk of progression to $\mathrm{MM}$, with free light chain (FLC) ratio, M-protein concentration, and depressed levels of non-affected immunoglobulins having been identified as risk factors for progression. ${ }^{1,3}$ Patients with MGUS have relatively few clonal plasma cells and tend to be asymptomatic. Some patients have a more active but still asymptomatic pre-malignant state called smoldering multiple myeloma (SMM), which has a higher yearly progression to MM. ${ }^{7,11}$ Finally, some patients initially present with a solitary plasmacytoma in a localized area of bone or, rarely, soft tissue but without evidence of widespread disease or end-organ damage; this population also has a high rate of progression to MM. ${ }^{12}$

\section{Clinical features}

MM may present with a variety of symptoms, the most common being bone pain and fatigue (Table 1). ${ }^{1,2,13}$ Easy bruising and bleeding, recurrent infection, weight loss, altered mental status, and other neurologic 
symptoms may also be present in the constellation of MM symptoms. Given that the clinical picture of $\mathrm{MM}$ is nebulous and nonspecific, it may be prudent to maintain an index of suspicion for MM in older adults with new onset back pain and unexplained anemia. ${ }^{14}$

\section{Hypercalcemia}

The manifestations of hypercalcemia range from mild to life-threatening and may include: altered mental status, fatigue, weight loss, nausea, vomiting, constipation, abdominal pain, and renal failure. ${ }^{2}$ The major cause of myelomatous hypercalcemia is thought to be widespread bone resorption. ${ }^{15}$

\section{Renal insufficiency}

The causes of renal failure in MM are multifactorial, with the main etiologies being monoclonal light chain deposition and hypercalcemia. Light chains may be observed on renal biopsy, in the form of protein-containing tubular casts. ${ }^{13}$ Renal insufficiency in MM is irreversible in $50 \%$ of cases. ${ }^{16}$ In rare instances, MM can lead to an acquired Fanconi syndrome. ${ }^{16}$

\section{Anemia}

Anemia is very common finding in MM. The anemia of MM tends to be normocytic and normochromic, though macrocytic anemia is also possible. ${ }^{2}$ Rouleaux formation is commonly seen on peripheral smear. ${ }^{13}$ Infrequently, MM may be associated with thrombocytopenia, though only very rarely do platelet counts fall below $20 \times 10^{9} / \mathrm{L}$.

\section{Infection}

Infection is a leading cause of morbidity and mortality in patients with MM. ${ }^{18}$ Immune deficiency in MM arises primarily due to deficiencies in normal gammaglobulins, but other elements of immune function are also compromised. Hypogammaglobulinemia is associated with infection by Streptococcus pneumoniae, Haemophilus influenzae, and other encapsulated bacteria. Treatment with corticosteroids, chemotherapy, and stem cell transplant also predispose patients to a variety of infections by additional Gram-positive (e.g., Staphylococcus aureus) and Gram-negative bacteria (e.g., Clostridium difficile, Escherichia coli), as well as viral (e.g., herpes simplex virus, varicella-zoster virus, cytomegalovirus, influenza), and fungal (e.g., Candida) pathogens. ${ }^{2,18}$

\section{Neurologic symptoms}

Up to $15 \%$ of patients with MM experience neurologic symptoms. ${ }^{2}$ The neurologic sequelae associated with MM can be varied, ranging from radiculopathy, spinal cord compression, cauda equina syndrome, cranial nerve palsies, and visual impairment, to the myriad of symptoms relating to hyperviscosity or hypercalcemia. Additionally, a number of MM chemotherapies have potential to cause permanent neuropathies. ${ }^{19}$

\section{Hyperviscosity}

Hyperviscosity is characterized by a classic triad of bleeding, visual disturbance, and focal neurologic signs. Bleeding tends to occur on mucosal surfaces, and may be due to M-protein inhibition of platelet function. ${ }^{20}$ Other evidence of end-organ dysfunction may be present, including high output cardiac failure. ${ }^{6,20}$ Hyperviscosity is mediated by large molecular size IgM paraproteins, while IgA and IgG paraproteins are smaller and less likely to lead to hyperviscosity symptoms; as such, hyperviscosity is far more commonly associated with Waldenstrom's macroglobulinemia than MM. ${ }^{21}$

Table I. Frequency of multiple myeloma features at time of diagnosis. ${ }^{1,7}$

\begin{tabular}{ll}
\hline Symptom & Frequency \\
\hline Bone pain & $58 \%$ \\
Fatigue & $32 \%$ \\
Weight loss & $24 \%$ \\
Anemia $(\mathrm{Hgb} \leq \mathrm{I} 20 \mathrm{~g} / \mathrm{L})$ & $73 \%$ \\
Hypercalcemia $($ serum Ca $\geq 2.75 \mathrm{mmol} / \mathrm{L})$ & $13 \%$ \\
Creatinine $\geq \mathrm{I} 77 \mu \mathrm{mol} / \mathrm{L}$ & $19 \%$ \\
\hline
\end{tabular}

Diagnostic criteria

The diagnosis of MM requires the presence of (1) clonal bone marrow plasma cells $\geq 10 \%$, or biopsy-proven plasmacytoma (bony or soft tissue); and (2) a myeloma-defining event, either (A) or (B):

A) Evidence of end-organ damage or dysfunction (known as CRAB features), not likely to be related to other medical conditions:

- Calcium: Serum calcium $\geq 2.75 \mathrm{mmol} / \mathrm{L}$, or $>0.25$ $\mathrm{mmol} / \mathrm{L}$ above normal limit;

- Renal failure: Serum creatinine $\geq 177 \mu \mathrm{mol} / \mathrm{mL}$ or creatinine clearance $<40 \mathrm{~mL} / \mathrm{min}$;

- Anemia: Hemoglobin $<100$ g/L, or $>20$ g/L below normal limit; or,

- Bone lesions: Any osteolytic lesions $\geq 5 \mathrm{~mm}$ on plain film radiography, computed tomography $(\mathrm{CT})$, or positron emission tomography (PET-CT).

- N.B., non-CRAB symptoms (e.g., infection, hyperviscosity) are considered non-specific and are not formally part of the diagnostic criteria for MM. 
B) Presence of biomarkers predicting progression to end-organ damage:

- Clonal bone marrow plasma cells $\geq 60 \%$;

- Involved/uninvolved FLC ratio $\geq 100$; or,

- Magnetic resonance imaging (MRI) showing $\geq 2$ focal lesions involving bone or marrow.

These diagnostic criteria reflect a 2014 revision by the International Myeloma Working Group, emphasizing the role of predictive biomarkers in order to encourage early detection and intervention before the onset of significant end-organ damage. ${ }^{6} \mathrm{MM}$ is now being diagnosed earlier than in the past, ${ }^{14}$ and evidence is emerging that early treatment may be associated with extended survival. ${ }^{22}$ However, it is important to note that treatment is not currently recommended for MGUS and SMM, so it remains important to exclude these possible diagnoses. ${ }^{7}$

\section{Investigations}

In practice, initial investigations for patients with clinical features suggestive of MM should include: complete blood count with differential; peripheral blood smear; serum electrolytes, including calcium (ionized, or corrected for albumin); lactate dehydrogenase; $\beta 2$-microglobulin $(\beta 2 \mathrm{M})$; albumin; serum and urine protein electrophoresis (SPEP, UPEP) and immunofixation; and urinalysis. ${ }^{24}$

The definitive diagnosis of MM requires either bone marrow aspiration or tissue biopsy to confirm presence of a plasmacytoma.

Full body radiographic skeletal survey has traditionally served to detect bony abnormalities, though low-dose whole-body CT is increasingly viewed as a reasonable and expeditious alternative. . $^{1,6-}$ 9,23 Additional PET-CT or whole-body MRI are now also recommended in patients with unclear bone involvement after skeletal survey. ${ }^{6,10}$

For prognostication, serum M-protein, serum FLC analysis, 24-hour urine protein, and light chain excretion, flow cytometry, or fluorescent in-situ hybridization (FISH) may be informative. ${ }^{2,10,14}$ Testing $\mathrm{M}$-protein isotype may be informative, as the rare IgD and IgE isotypes are associated with poor prognosis. ${ }^{7,24}$ Elevated lactate dehydrogenase is indicative of advanced disease, and is another poor prognostic indicator. ${ }^{24}$

Plasma cell labeling index (PCLI) is a measure of plasma cell proliferation. It is a highly labour intensive technique so it is not commonly seen in clinical use, despite its good prognostic value. ${ }^{24}$

A detailed discussion of genetic investigations is beyond the scope of this review, but conventional cytogenetics, FISH, and gene expression profiling (GEP) can be used in risk stratification. ${ }^{24}$ Conventional cytogenetic approaches are less often used than in the past, given their low sensitivity for karyotypic abnormalities. FISH does not require actively proliferating cells to detect abnormalities, and is therefore a more sensitive technique. GEP is a developing technology examining transcriptional activity of MM cells. Some studies indicate that GEP may improve risk stratification, but it has yet to be adopted in clinical settings. ${ }^{24}$

\section{Staging}

MM is currently staged using the Durie-Salmon Staging System (DSS) and the International Staging System (ISS), with ISS currently being favoured for its simplicity and objectivity. ${ }^{25}$ However, neither system entirely accounts for the heterogeneity of MM disease course.

DSS is an estimate of MM tumor burden, based on measures of clinical, laboratory, and radiographic features of end-organ damage. While this system has better prognostic value than other historical staging systems (e.g., Merlini-Waldenstrom-Jayakar staging system, Medical Research Council staging system), the need for subjective interpretation of lytic bone lesions on skeletal survey can limit the reliability of DSS. ${ }^{25}$

Since its introduction in 2005, ISS has become the more widely used staging system, involving only two objective and readily reproducible measurements, specifically $\beta 2 \mathrm{M}$ and albumin (Table 2 ). The ISS stages correlate fairly well with DSS, but has more uniform distribution of patients across its three stages. ${ }^{25,26}$ Unfortunately, ISS is only validated for use in patients with symptomatic MM, and it has unclear utility for MGUS, SMM, or other plasma cell disorders. ${ }^{5}$ Moreover, ISS does not necessarily reflect tumor burden since $\beta 2 \mathrm{M}$ can be elevated secondary to renal failure unrelated to MM.

\begin{tabular}{ll} 
Table 2. International staging system criteria \\
\hline Stage & Criteria \\
\hline ISS I & Albumin $\geq 35 \mathrm{~g} / \mathrm{L}$, and \\
& $\beta_{2} \mathrm{M}<3.5 \mathrm{mg} / \mathrm{L}$ \\
ISS II & Not stage I or III \\
ISS III & $\beta_{2} \mathrm{M} \geq 5.5 \mathrm{mg} / \mathrm{L}$, regardless of albumin \\
\hline
\end{tabular}

Unfortunately, while these staging systems are useful for prognostication, they are of little guidance when making treatment choices for MM. ${ }^{7}$ As new and more effective therapeutics are now being introduced, it is unclear whether these staging systems will remain useful in years to come,$^{5}$ though limited data suggest that ISS may continue to have prognostic value even in 
the advent of novel agents. ${ }^{27}$

The Mayo Stratification of Myeloma and Risk-Adapted Therapy (mSMART) is a predictive system developed by the Mayo Clinic, which incorporates genetic data from conventional cytogenetics, FISH, and GEP (Table 3). ${ }^{7,24}$ It has shown encouraging prognostic value in newly-diagnosed MM patients taking novel agents, but has not been validated in prospective studies, and is not currently used in clinical practice. ${ }^{24}$

Table 3. Mayo stratification of myeloma and risk-adapted therapy (mSMART) criteria

\begin{tabular}{ll}
\hline High Risk & FISH: del(I7p), $\mathrm{t}(|4 ;| 6), \mathrm{t}(\mid 4 ; 20)$ \\
& GEP: high risk signature \\
Intermediate & FISH: $\mathrm{t}(4, \mid 4)$ \\
Risk & Cytogenetic del(I3) \\
& Hypodiploidy \\
& PCLI $\geq 3 \%$ \\
Standard Risk & All others, including: \\
& FISH: $\mathrm{t}(\mathrm{II} ; \mathrm{I}), \mathrm{t}(6 ; \mid 4)$ \\
\hline
\end{tabular}

\section{Treatment}

The treatment of MM is complex and continues to evolve, and so a detailed discussion of treatment regimens is beyond the scope of this review. However, there are a number of treatment guidelines and algorithms available. ${ }^{7,23,28-30}$

Broadly speaking, the stages of treatment may be described as initiation and consolidation/maintenance. Several multi-drug regimens are employed in the initiation stage, commonly involving combinations of: dexamethasone, lenalidomide, bortezomib, thalidomide, and cyclophosphamide. Younger patients with a favourable functional status may enjoy improved survival with autologous stem cell transplant (ASCT), though the optimal timing for this intervention is still unclear. ${ }^{18,31}$ Maintenance therapy tends to be more streamlined than during the initiation stage, with lenalidomide or bortezomib often used as single agents during maintenance. There is much interest in the study of new regimens for use against MM relapse. ${ }^{31}$

From a historical perspective, the introduction of alkylating agents (e.g., melphalan) improved median survival in MM from less than one year to 2.5 years; along with corticosteroid therapy, these agents served as the traditional mainstays of multiple myeloma treatment. ${ }^{2,32}$ The past two decades have seen significant advances in MM treatment; in the current era of ASCT, thalidomide, lenalidomide, and the proteasome inhibitor bortezomib median survival has increased to over four years. ${ }^{33}$

Although MM remains an incurable malignancy, new agents in development promise to further improve patient survival. ${ }^{19,31,33,34}$ Monoclonal antibodies (daratumumab, elotuzumab) and next-generation proteasome inhibitors (carfilzomib, ixazomib, marizomib) are currently being investigated. Additional agents in development seek to target alternative pathways in MM pathogenesis (e.g., signal transduction, heat-shock proteins, epigenetic modulation).

In addition to active treatment, the prevention and management of MM complications (e.g., hypercalcemia, renal insufficiency, infection, fractures, hyperviscosity) is also an essential component of MM care. ${ }^{5,20,31}$ There is evidence for the use of bisphosphonates for reducing pathologic fracture risk and perhaps improving survival in $\mathrm{MM}^{35}$ The evidence for antibiotics for infection prophylaxis in MM is equivocal. ${ }^{21,35}$ Antibiotic therapy may need to be tailored according to renal function. It is advisable for patients to be vaccinated against influenza, and patients receiving certain treatment regimens may benefit from antiviral prophylaxis against herpes zoster. ${ }^{6,23}$

\section{Conclusion}

MM is a hematologic malignancy that can present with a variety of non-specific symptoms. ${ }^{2,14}$ It is primarily a disease of the elderly, and many patients will exhibit bone pain, incidental bone lesions, and anemia. The cause of MM is unknown and there is no cure at present, though treatment regimens continue to become more sophisticated. The diagnosis of MM requires a bone marrow biopsy, or plasmacytoma biopsy, and it is important to exclude MGUS and SMM because these conditions do not require treatment. ${ }^{6,22}$

\section{References}

1. Kyle RA, Gertz MA, Witzig TE, et al. Review of 1027 patients with newly diagnosed multiple myeloma. Mayo Clinic Proceedings 2003;78(1):21-33.

2. Gupta M, Pal R, Tikoo D. Multiple myeloma: the disease and its treatment. Int J Basic Clin Pharmacol 2013;2(2):103.

3. Kyle RA, Rajkumar SV. Multiple Myeloma. N Engl J Med 2004;351(18):1860-73.

4. Alexander DD, Mink PJ, Adami H-O, et al. Multiple myeloma: a review of the epidemiologic literature. Int $\mathrm{J}$ Cancer 2007;120(S12):40-61.

5. Kyle RA, Rajkumar SV. Criteria for diagnosis, staging, risk stratification and response assessment of multiple myeloma. Leukemia 2008;23(1):3-9.

6. Rajkumar SV, Dimopoulos MA, Palumbo A, et al. International Myeloma Working Group updated criteria for the diagnosis of multiple myeloma. The Lancet Oncology 2014;15(12):e538-48.

7. Rajkumar SV. Multiple myeloma: 2014 update on diagnosis, risk-stratification, and management. Am J Hematol 2014;89(10):998-1009.

8. Canadian Cancer Society's Advisory Committee on Cancer Statistics. Canadian Cancer Statistics - 2015. Toronto, ON: Canadian Cancer Society;2015.

9. Becker N. Epidemiology of Multiple Myeloma. In: Recent Results in Cancer Research. Vol 183. Recent Results in Cancer 
Research. Berlin, Heidelberg: Springer Berlin Heidelberg; 2011:25-35.

10. Raab MS, Podar K, Breitkreutz I, Richardson PG, Anderson KC. Multiple myeloma. The Lancet 2009;374(9686):324-39.

11. Rajkumar SV, Landgren O, Mateos MV. Smoldering multiple myeloma. Blood 2015;125(20):3069-75.

12. Dingli D, Kapoor P. Solitary Plasmacytoma. In: Multiple Myeloma. New York, NY: Springer New York;2013:195-210.

13. Dispenzieri A, Kyle RA. Multiple myeloma: clinical features and indications for therapy. Best Pract Res Cl Ha 2005;18(4):553-68.

14. Röllig C, Knop S, Bornhäuser M. Multiple myeloma. Lancet. December 2014. doi:10.1016/S0140-6736(14)60493-1.

15. Oyajobi BO. Multiple myeloma/hypercalcemia. Arthritis Res Ther 2007.

16. Bladé J, Rosiñol L. Renal, hematologic and infectious complications in multiple myeloma. Best Pract Res Clin $\mathrm{Ha}$ 2005;18(4):635-52.

17. Angtuaco EJC, Fassas ABT, Walker R, Sethi R, Barlogie B. Multiple Myeloma: clinical review and diagnostic imaging. Radiology 2004;231(1):11-23.

18. Nucci M, Anaissie E. Infections in patients with multiple myeloma in the era of high-dose therapy and novel agents. Clin Infect Dis 2009;49(8):1211-25.

19. Dispenzieri A, Kyle RA. Neurological aspects of multiple myeloma and related disorders. Best Pract Res Clin Ha 2005;18(4):673-88.

20. Adams BD, Baker R, Lopez JA, Spencer S. Myeloproliferative disorders and the hyperviscosity syndrome. Hematol Oncol Clin North Am 2010;24(3):585-602.

21. Kwaan HC. Hyperviscosity in plasma cell dyscrasias. Clin Hemorheol Microcirc 2013;55(1):75-83.

22. Mateos M-V, Hernández M-T, Giraldo $\mathrm{P}$, et al. Lenalidomide plus dexamethasone for high-risk smoldering multiple myeloma. N Engl J Med 2013;369(5):438-47.

23. Terpos E, Kleber M, Engelhardt M, et al. European myeloma network guidelines for the management of multiple myeloma-related complications. Haematologica 2015;100(10):125466.
24. van de Donk NWCJ, Sonnevald P. Diagnosis and risk stratification in multiple myeloma. Hematol Oncol Clin North Am 2014;28(5):791-813.

25. Roy V, Greipp PR. Staging of Multiple Myeloma. In: Multiple Myeloma. New York, NY: Springer New York; 2013:47-53.

26. Greipp PR, Miguel JS, Durie BGM, et al. International staging system for multiple myeloma. J Clin Oncol 2005;23(15):341220.

27. Kastritis E, Zervas K, Symeonidis A, et al. Improved survival of patients with multiple myeloma after the introduction of novel agents and the applicability of the International Staging System (ISS): an analysis of the Greek Myeloma Study Group (GMSG). Leukemia 2009;23(6):1152-7.

28. Engelhardt M, Terpos E, Kleber M, et al. European Myeloma Network recommendations on the evaluation and treatment of newly diagnosed patients with multiple myeloma. Haematologica 2014;99(2):232-42.

29. Ludwig H, Miguel JS, Dimopoulos MA, et al. International Myeloma Working Group recommendations for global myeloma care. Leukemia 2014;28(5):981-92.

30. Pratt G, Jenner M, Owen R, Snowden JA. Updates to the guidelines for the diagnosis and management of multiple myeloma. Brit J Haematol 2014;167(1):131-3.

31. Rajkumar SV, Kumar S. Multiple Myeloma: diagnosis and treatment. Mayo Clin Proc 2016;91(1):101-19.

32. Kumar SK, Rajkumar SV, Dispenzieri A, et al. Improved survival in multiple myeloma and the impact of novel therapies. Blood 2008;111(5):2516-20.

33. Ludwig H, Sonneveld P, Davies F, et al. European perspective on multiple myeloma treatment strategies in 2014. Oncologist 2014;19(8):829-44.

34. Mimura N, Hideshima T, Anderson KC. Novel therapeutic strategies for multiple myeloma. Exp Hematol 2015;43(8):73241.

35. Raje NS, Yee AJ, Roodman GD. Advances in supportive care for multiple myeloma. J Natl Compr Canc Netw 2014;12(4):502-11.

\title{
DiAGNOSE THIS
}

\section{A 43-year-old female with new onset vertigo}

\author{
Rebecca George BSc ${ }^{1}$, Emad Massoud MB, MSc, FRCSC ${ }^{2}$ \\ ${ }^{1}$ Class of 2017, Faculty of Medicine, Dalhousie University \\ ${ }^{2}$ Division of Otolaryngology - Head and Neck Surgery, Department of Surgery, \\ Dalhousie University
}

\begin{abstract}
43 year-old female presents to the emergency Adepartment with a three-day history of vertigo with nausea and vomiting. She states that the onset was sudden and severe, and denies any history of similar incidents. She does not report any headache and has not noticed a change in her hearing or the presence of tinnitus. Neurologic exam was unremarkable, but she displayed gait instability to the left side. There is a horizontal right beating nystagmus present. Bedside caloric test reveals reduced response in her left ear.
\end{abstract}

What is the most likely diagnosis?

A. Isolated inferior cerebellar stroke B. Episode of benign paroxysmal positioning vertigo

C. Vestibular neuronitis

D. Multiple sclerosis

E. Ménière's disease 